\title{
Semantic and plausibility effects on preview benefit during eye fixations in Chinese reading
}

\author{
Jinmian Yang $\cdot$ Suiping Wang $\cdot$ Xiuhong Tong • \\ Keith Rayner
}

Published online: 18 November 2010

(C) The Author(s) 2010. This article is published with open access at Springerlink.com

\begin{abstract}
The boundary paradigm (Rayner, 1975) was used to examine whether high level information affects preview benefit during Chinese reading. In two experiments, readers read sentences with a 1-character target word while their eye movements were monitored. In Experiment 1, the semantic relatedness between the target word and the preview word was manipulated so that there were semantically related and unrelated preview words, both of which were not plausible in the sentence context. No significant differences between these two preview conditions were found, indicating no effect of semantic preview. In Experiment 2, we further examined semantic preview effects with plausible preview words. There were four types of previews: identical, related \& plausible, unrelated \& plausible, and unrelated \& implausible. The results revealed a significant effect of plausibility as single fixation and gaze duration on the target region were shorter in the two plausible conditions than in the implausible condition. Moreover, there was some evidence for a semantic preview benefit as single fixation duration on the target region was shorter in the related \& plausible condition than the unrelated \& plausible condition. Implications of these results for processing of high level information during Chinese reading are discussed.
\end{abstract}

Keywords Eye-movements · Reading Chinese - Preview benefit · Semantic and plausibility effects

\footnotetext{
J. Yang $(\square) \cdot$ K. Rayner

Department of Psychology, University of California, San Diego, San Diego, CA 92092, USA

e-mail: jinmianyang@ucsd.edu

S. Wang $\cdot \mathrm{X}$. Tong

Department of Psychology, South China Normal University, Guangzhou, China
} 
There is no doubt that readers extract useful information from the word they are looking at (i.e., the foveal word). Moreover, it has been confirmed by a large number of studies that readers obtain preview information from the word to the right of fixation (i.e., the parafoveal word) and then use it to identify that word when it is later fixated (see Rayner, 1998, 2009 for reviews). This facilitation has been referred to as parafoveal preview benefit, and it has typically been assessed via the use of a gaze-contingent boundary paradigm (Rayner, 1975). In experiments of this sort, an invisible, predetermined boundary is placed just to the left of a target word location, which is initially occupied by a preview word. When the reader's eyes cross the boundary location, the preview word is replaced by the target word. Since this display change occurs during a saccade, when vision is suppressed, readers generally do not notice it. With this paradigm, the kind of information conveyed by the preview word can be controlled and varied. Parafoveal preview benefit is defined as the amount of time that readers look at the target word when given a valid preview subtracted from the amount of time that they look it when they didn't have a valid preview.

There have generally been convergent findings regarding the nature of parafoveal preview benefit during the reading of alphabetic languages. That is, readers obtain sub-lexical information from the parafovea, such as orthographic or partial word information (Lima \& Inhoff, 1985; Lima, 1987; Inhoff, 1989, 1990; Briihl \& Inhoff, 1995; Rayner, 1975), word length information (Morris, Rayner, \& Pollatsek, 1990; Inhoff, Radach, Eiter, \& Juhasz, 2003; Juhasz, White, Liversedge, \& Rayner, 2008) and phonological information (Pollatsek, Lesch, Morris, \& Rayner, 1992; Henderson, Dixon, Petersen, Twilley, \& Ferreira, 1995; Ashby \& Rayner, 2004; Ashby, Treiman, Kessler, \& Rayner, 2006; Chace, Well, \& Rayner, 2005; Miellet \& Sparrow, 2004), and use it to identify the word when it is fixated. However, parafoveal preview benefits do not seem to extend to semantic information (Altarriba, Kambe, Pollatsek, \& Rayner, 2001; Balota, Pollatsek, \& Rayner, 1985; Hyönä \& Häikiö, 2005; Rayner, Balota, \& Pollatsek, 1986; Rayner \& Morris, 1992). For example, Rayner et al. (1986) presented readers with four types of parafoveal previews with respect to a target word (in the example used here, tune): identical (tune), orthographically similar (turc), semantically related (song), or semantically unrelated (door). They found parafoveal preview benefit in the identical and orthographically similar conditions, but there was no difference between the semantically related and unrelated previews. More recently, Altarriba et al. (2001) used fluent Spanish-English bilinguals to study parafoveal semantic processing in reading. Target words could be English or Spanish words and their previews were translations of the other language. There were five kinds of previews: identical (cream as a preview for cream), cognate translations (crema was a preview for cream), non-cognate translations (fuerte, which means strong, was a preview for strong), pseudocognates (words that are unrelated except that they are orthographically similar such as grasa as a preview for grass), or unrelated words (grito as a preview for sweet). There was no preview benefit from non-cognate translations, and preview benefit from cognates did not differ from that of pseudo-cognates. These findings 
again indicate that preview benefit is due to orthographic overlap of previews and targets, but not to semantic relatedness. ${ }^{1}$

The failure to observe semantic parafoveal preview benefit in reading English does not mean that semantic information isn't obtained from the parafovea. The fact that one third of all words are skipped during reading (because they were identified when they were in the parafovea) implies that semantic information can be gleaned from the parafovea. However, if this information is not sufficient for identifying the word, it will be of little benefit in lexical access when the word is fixated. That is, semantic information is not integrated across fixations in reading English. Thus, although readers can obtain semantic information from the parafovea (and when they do, they skip the word), this does not equate to semantic parafoveal preview benefit (Rayner, 1998, 2009).

Compared to alphabetic languages, less is known about the nature of parafoveal preview benefit with respect to Chinese, a logographic writing system that is drastically different from alphabetic scripts in how meaning and speech are represented (Yang, Wang, Chen, \& Rayner, 2009; Wang, Chen, Yang, \& Mo, 2008). So far, most of the studies examining parafoveal preview benefit during the reading of Chinese have focused on low level information, such as phonological and orthographic information. For example, Liu, Inhoff, Ye, and Wu (2002) found that Chinese readers spent less time on a target word when it was preceded by a phonologically or orthographically similar preview word. A similar phonological preview benefit was observed by Tsai, Lee, Tzeng, Hung, and Yen (2004). These findings suggest that phonological and orthographic preview benefits are common across English and Chinese. However, regarding semantic parafoveal preview benefit, the null effects from alphabetic languages may not be generalizable to Chinese given its particular properties, which will be elaborated below.

First, unlike alphabetic writing systems, written Chinese is formed by strings of equally spaced box-like symbols called characters. Basically, there are many visual details packed into characters, since they can differ in the number of strokes and the manner of construction. There are two groups of Chinese characters: integrated characters and compound characters. The integrated characters consist of crossed strokes that are inseparable, whereas compound characters usually consist of two separable subcomponents that denote semantic or phonological information (called radicals). Given that information is more densely packed in Chinese than English, more information may be visible to the right of fixation in Chinese compared to alphabetic writing systems. This hypothesis is supported by the finding that in Chinese the size of the perceptual span to the right of fixation (2-3 characters) is only slightly larger than the average size of forward saccades (2.6 characters), indicating that there is only slight overlap in the perceptual span in reading Chinese (Inhoff \& Liu, 1998). On the other hand, there is considerable overlap (up to 50\%) between the right-side area of successive spans in reading English (Rayner, 1998); the perceptual span to the right of a fixation (about 14-15 letter spaces) is about twice the size of the average forward saccade (7-8 letter spaces). This implies that

\footnotetext{
${ }^{1}$ There is some evidence for semantic preprocessing of previews within Finnish compound words (White, Bertram, \& Hyönä, 2008).
} 
Chinese readers are able to obtain the maximum amount of new information from the right of fixation on each fixation (Chen, Song, Lau, Wong, \& Tan, 2003).

Second, Chinese characters more closely map to meaning than to sound. While in English and other alphabetic languages, the mapping between orthography and phonology is relatively transparent, the pronunciation of a Chinese character is largely opaque. Moreover, the pronunciation of each character is monosyllabic, and there are only about 1,300 different syllables used in Mandarin Chinese. Given that there are as many as 87,019 characters (about 5,000 of them are commonly used), there are a lot of homophone pairs (Yin, 1984). Hence, phonological mediation in lexical access in Chinese could be less efficient than that in alphabetic languages, and access of meaning may be more likely to go directly from orthography (e.g., Chen, Flores d'Arcais, \& Cheung 1995; Chen \& Shu, 2001). Since phonological preview benefit was observed during Chinese reading (Liu et al., 2002, Tsai et al., 2004), there is therefore a good chance that semantic preview benefit might also exist.

Finally, while words are regarded as the visually distinctive units in alphabetic languages, characters are regarded as the visually distinctive units in written Chinese. Although one character can be a single-character word by itself, most characters can join with other characters to form a multiple-character word, with the meaning different in these two situations. In addition, there is no physical mark between words (the width of the space between words is identical to that between characters within a word). Thus, readers have to rely on context to determine whether a character is a word by itself or if it is a morpheme of a multiple-character word, which means that readers may need to obtain more information from the right of fixation to comprehend the text on-line.

In short, despite the finding that there is little evidence for a semantic parafoveal preview effect during the reading of alphabetic languages, this is not necessarily the case during Chinese reading. Indeed, Yan, Richter, Shu, and Kliegl (2009) recently reported semantic preview benefit for integrated characters (which were referred as pictographical or indicative characters) during the reading of Chinese sentences. Nevertheless, given that about $82 \%$ of all characters are compound characters in Chinese (Xu, Pollatsek, \& Potter, 1999), whether or not the semantic preview benefit observed in Yan et al.'s experiment is common is still an open question. Therefore, in the experiments reported here, we used both integrated and compound characters as targets (the majority of which were compound characters) to address this issue. Furthermore, we examined evidence for semantic preview benefit in two ways: (1) by manipulating the semantic relation between the target word and the preview word (Experiments 1 and 2), and (2) by manipulating the plausibility of the preview word (Experiment 2). By plausibility, we mean the extent to which a given target word represents a likely (plausible) or unlikely (implausible) event described in a sentence. Plausibility differs from predictability in that the latter typically refers to how well participants can predict a specific target word, whereas the former term refers to how likely a target word represents a real world event. A plausible target word could be either a high or low predictable word in a sentence, but an implausible word will always be unpredictable. We shall provide more explanation for the plausibility manipulation in Experiment 2. 


\section{Experiment 1}

In Experiment 1, single character words, which allowed us to manipulate the meaning of previews within a perceptual unit, were used as target words. The semantic relation between the target words and the previews was manipulated such that there were three types of previews: (1) the preview and the target word were identical, (2) the preview was semantically related to the target word, and (3) the preview was semantically unrelated to the target word. Based on prior research (Inhoff \& Liu, 1997, 1998; Liu et al., 2002; Yang et al., 2009a, b), we expected that reading times on the target word in the identical preview condition would be shorter than the other two preview conditions. The more critical question was whether or not the reading time on the target word would be shorter when it was preceded by a semantically related preview than a semantically unrelated preview.

\section{Method}

Participants. Twenty-four undergraduate students from South China Normal University, who were all naïve concerning the purpose of the experiment, participated for course credit. They all had normal or corrected to normal vision and were native readers of Chinese.

Materials. Fifty-four single-character words were chosen as target words, each of them was paired with two types of single-character preview words: semantically related and semantically unrelated, which will be referred to as related and unrelated previews, respectively. All of the target and preview words were nouns and were embedded into 54 experimental sentences which were 11-20 characters in length. The target words were located in the middle of the sentence with at least 4 characters ahead or following and there was no punctuation until the end of the sentence. An example sentence (with the target word, related and unrelated preview words in bold) and English translation is:

\section{1. 医生叫他尝试睁开眼/脸/院看远处的灯光。}

(The doctor encouraged him to open his eyes/face/yard to look at the light over there.)

Word frequency and number of strokes were closely matched between target words and their corresponding preview words. The frequencies of occurrence for the target, its related preview, and its unrelated preview averaged $370(\mathrm{SD}=482), 426$ $(\mathrm{SD}=593)$, and $457(\mathrm{SD}=752)$ per million, ${ }^{2}$ with $\log$ mean frequencies of 2.18 , 2.20 , and 2.11, respectively; $F<1$. The number of strokes averaged 9.2 $(\mathrm{SD}=3.1)$, $8.5(\mathrm{SD}=3.7)$, and $8.9(\mathrm{SD}=3.1)$ for these conditions, $F(2,106)=1.9, p>.10$. Moreover, to avoid any orthographic and homophonic benefit from the related and unrelated previews, such similarities between the target and these two previews were controlled. That is, the related and unrelated previews did not share any radicals (component of Chinese characters) and syllables with the target.

\footnotetext{
${ }^{2}$ The word frequency counts are based on a Chinese Dictionary (China Daily, 1998).
} 
The eye movement contingent boundary technique (Rayner, 1975) was used to present different previews (identical, related, and unrelated) that changed to the target word when the reader moved his/her eyes across an invisible boundary that was set at the end of character $n-1$. Three counterbalanced material sets were created, each containing 54 experimental sentences and 30 filler sentences. The experimental stimuli in each set included 18 sentences in each of three preview conditions. Each version of the experimental sentences appeared once across the three sets.

Rating Studies. A semantic relatedness rating study was conducted in which participants were asked to rate the semantic relatedness between the target words and each of their preview words (related and unrelated) on a 5-point scale (where $1=$ unrelated; $5=$ highly related). Two counterbalanced material sets were created, each containing 27 pairs of target-related preview words and 27 pairs of targetunrelated preview words. Sixteen students were randomly assigned to one of these lists. The results revealed that semantic relatedness between the related preview words and the target words $(M=3.83, \mathrm{SD}=.75)$ was significantly stronger than that between the unrelated preview words and the target words $(M=1.87$, $\mathrm{SD}=.62), t 1(15)=11.5, p<.001, t 2(53)=29.8, p<.001$.

In addition, a plausibility rating study was conducted to evaluate how well the target word, related, and unrelated preview word fit into the sentence. Since only the first part of the sentences is available for readers to judge the plausibility of the target word or its preview word, this norming was done on the first part of the sentences. Eighteen participants were presented the first part of the sentences up to (and including) the target word or its preview words and were asked to rate the plausibility of the sentence (assuming that the sentence will end with a second part) on a 5 -point scale (where $1=$ highly implausible; $5=$ highly plausible). Thus, there were three versions of sentences, and three counterbalanced material sets were made by including a third of the sentences in each version. The results showed that there was a significant effect of plausibility, $F 1(2,34)=53.90, \mathrm{p}<.001, F 2$ $(2,106)=168.3, p<.001$. Sentences with the target words were rated more plausible $(M=3.88, \mathrm{SD}=.74)$ than those with related previews $(M=2.32$, $\mathrm{SD}=.67)$, and those with unrelated previews $(M=2.13, \mathrm{SD}=.53)$, $t \mathrm{~s}>7.0$, $p s<.001$. There was no significant difference between the later two conditions, $p s>.1$.

Furthermore, a cloze predictability norming study was conducted to examine how often readers could predict the target words or their preview words from the prior context. Ten undergraduate students were given the first part of the experimental sentence up to (and including) the character to the left of the target word and asked to provide the next word in the sentence (i.e., predict the target word). $24 \%$ of the time participants predicted the target word and they never predicted the related and unrelated preview words.

All participants in the above rating studies were undergraduate students from the South China Normal University. They did not participate in the eye-tracking portion of the experiment, and they only participated in one of these rating studies.

Apparatus. An SR Eyelink 1000 eye-tracking system was used to track readers' eye movements at a rate of $1,000 \mathrm{HZ}$. The eye-tracker monitored movements of the 
right eye, although viewing was binocular. A Dell 19-in. SVGA monitor was used to display the stimuli. The monitor was set to a refresh rate of $150 \mathrm{~Hz}$. The delay in detecting an eye movement crossing the boundary and changing the display averaged $9 \mathrm{~ms}$. All stimuli were presented in white on a black background on the computer monitor. All characters were printed in simple Kai font. Each character was about $1.0 \times 1.0 \mathrm{~cm}$ in size and subtended approximately 1.2 degrees of visual angle (with the participants' eyes being $57 \mathrm{~cm}$ away from the monitor).

Procedure. Prior to beginning the experiment, participants were given the experimental instructions. They were randomly assigned to one of three stimulus sets and were tested individually. The experiment consisted of a calibration phase and an experimental phase. In the calibration phase, each participant performed a 3 -point calibration procedure to make sure that the eye-tracker recordings were accurate. The experimental phase then followed.

At the beginning of the experimental phase, participants were asked to read each sentence carefully for comprehension. Before reading each sentence, they were first asked to fixate on a dot in the center of the computer screen and then a box on the left side of the screen that coincided with the position of the first character of the sentence. Once they fixated on the box, the sentence was displayed. One-third of the sentences were followed by a true-false comprehension question. Each participant read the 54 experimental and 30 filler sentences in a random order; the experiment lasted about $30 \mathrm{~min}$. Nine practice sentences were presented at the beginning of the experiment to familiarize participants with the procedure; they were informed that they could take a break whenever they needed one.

\section{Results}

All participants averaged over $80 \%$ correct on the comprehension questions (mean $=89 \%$ ). Fixations less than $60 \mathrm{~ms}$ or greater than $600 \mathrm{~ms}$ were eliminated from the analyses. Trials in which the display change occurred during a fixation were also excluded. In total $10.4 \%$ of the data were lost, including track losses (there were no differences in amount of data loss across conditions).

Two sets of analyses were carried out on the eye movement data. The first set was based on individual characters, whereas the second was based on regions (i.e., multiple characters). Analyses were performed using a linear mixed-effects (lme) model specifying participants and items as crossed random effects. These analyses were carried out using the lmer4 package (Bates \& Maecher, Bates and Maechler 2009) in R, an open-source programming language and environment for statistical computation (R Development Core Team, 2009). Two contrasts were set up: (1) the identical preview versus the average of the related and unrelated previews (to examine the typical preview benefit), and (2) the related versus unrelated preview (to examine semantic preview benefit). We report regression coefficients ( $b$ s, effects relative to the intercept, which indicate effect size in milliseconds), standard errors $(S E \mathrm{~s})$, and $p$ values estimated using posterior distributions for model parameters obtained by Markov Chain Monte Carlo sampling (Baayen, 2008, Baayen, Davidson, \& Bates, 2008). 


\section{Character-based Analysis}

We computed three early eye movement measures, which are typically used in studies of parafoveal preview effects (Rayner, 1998), associated with characters $n-1$ through character $n+1$ (relative to the target character $n$ ) as a function of preview type (see Table 1). These measures were the probability of initially skipping the character, single fixation duration (i.e., the character was fixated exactly once on the first pass reading through the text), ${ }^{3}$ and gaze duration (i.e., the sum of all fixations on a character prior to moving to another character). The means and standard deviations of these measures are shown in Table 1. We will not discuss skipping probability further as all differences across the different conditions were quite small and unreliable.

Character $n-1$. The reason we were interested in the effect of the preview manipulation on this character is because it has been reported that the processing of the parafoveal word ${ }^{4}$ can sometimes exert an influence on the currently fixated word (a parafoveal-on-foveal effect) for readers of Chinese (Yang et al., 2009a, Experiment 1, with 1-character words as targets). However, neither contrast was significant in single fixation duration or gaze duration on this character $(p s>.1)$. It appears that parafoveal-on-foveal effects in reading Chinese, as in reading English, are not fully reliable and are not easy to replicate across studies (see Rayner \& Juhasz, 2004; Rayner, White, Kambe, Miller, \& Liversedge, 2003; Starr \& Rayner, 2001 for reviews).

Target word. Single fixation duration in the identical preview condition was $26 \mathrm{~ms}$ shorter than the average of the related and unrelated conditions, $b=19$, $\mathrm{SE}=5.0, p<.001$. However, there was no significant difference between the later two conditions, $b=5, \mathrm{SE}=8.9, p>.5$. A similar pattern was found in the gaze duration data wherein readers' fixations were shorter in the identical condition than the average of the related and unrelated condition, $b=21$, SE $=5.5, p<.001$, with no significant difference between the later two conditions, $b=5$, SE $=5.0, p>.6$.

Character $n+1$. The influence of different preview characters was evident in single fixation duration, which was shorter in the identical condition than the average of the other two conditions, $b=9, \mathrm{SE}=4.5, p<.05$; however, the difference between the unrelated and related condition was again not significant, $p>.1$. There were no significant effects in gaze duration, $p \mathrm{~s}>.1$.

\section{Region-based Analysis}

While the character based analysis is informative, it is the case that there were considerable missing data due to the high skipping rates for individual Chinese

\footnotetext{
${ }^{3}$ We also computed the first fixation duration (i.e., the duration of the first fixation on a character independent of the number of fixations on this character), which showed a similar pattern to single fixation duration.

4 The word to the right of fixation in Chinese may not technically be in the parafovea, but for consistency with prior research, we will use the term "parafoveal word".
} 
Table 1 Character-based analysis: means of eye movement measures by participants for character $n-1$ to character $n+1$ in Experiment 1

Values in parentheses represent standard deviations

\begin{tabular}{lccc}
\hline & Identical & Related & Unrelated \\
\hline $\mathrm{n}-1$ & & & \\
Skipping & $.56(.16)$ & $.59(.16)$ & $.57(.13)$ \\
Single fixation (ms) & $219(30)$ & $223(39)$ & $229(49)$ \\
Gaze duration (ms) & $222(31)$ & $225(39)$ & $231(46)$ \\
$\mathrm{n}$ (target word) & & & \\
Skipping & $.43(.19)$ & $.42(.20)$ & $.44(.17)$ \\
Single fixation (ms) & $240(39)$ & $265(47)$ & $268(51)$ \\
Gaze duration (ms) & $248(45)$ & $279(57)$ & $278(52)$ \\
$\mathrm{n}+1$ & & & \\
Skipping & $.55(.15)$ & $.56(.15)$ & $.52(.12)$ \\
Single fixation (ms) & $224(31)$ & $238(39)$ & $243(48)$ \\
Gaze duration (ms) & $245(91)$ & $240(36)$ & $245(43)$ \\
\hline
\end{tabular}

characters (about 50\% in first pass reading), and the results could be easily affected by mislocalized fixations (where readers intended to fixate on character $n$, for example, but actually landed on character $n-1$, see Drieghe, Rayner, \& Pollatsek, 2008). We therefore computed a region based analysis in which different characters were combined as a region (so as to reduce the skipping rate). Specifically, characters $n-1$ and $n-2$ were combined as a pre-target region $\mathrm{T}-1$, and characters $\mathrm{n}$ and $\mathrm{n}+1$ were combined as a target region $\mathrm{T}$. Since region $\mathrm{T}-1$ was prior to the boundary location, there was a preview word in the target location when the eyes fixated in this region. When the eyes crossed the boundary and landed on region $\mathrm{T}$, the preview was replaced by the target word in all preview conditions.

Single fixation duration and gaze duration ${ }^{5}$ were computed for region $\mathrm{T}-1$ and T. As in the character-based analysis, the probability of initially skipping, which was reduced to about $17 \%$, will not be discussed further because no significant differences were observed across the conditions. However, for completeness, they are shown in Table 2.

Pre-target Region. The effect of the preview manipulation was not significant for either single fixation duration or gaze duration, $p \mathrm{~s}>.5$.

Target Region. As in the character-based analysis, significant differences emerged between the identical condition and the average of the other two preview conditions: for single fixation duration, $b=16$, $\mathrm{SE}=4.7, p<.001$, for gaze

\footnotetext{
5 When regions larger than a single word are examined, the measure is usually referred to as first pass reading time (the sum of all fixations in a region before leaving the region, see Rayner, 1998, 2009). While the regions analyzed in the present experiments are technically larger than a single word, for simplicity we will use the term gaze duration to refer to the sum of all fixations in the region before moving to another region.
} 
Table 2 Region-based analysis: Means of eye movement measures by participants for the pre-target and target region in Experiment 1

Values in parentheses represent standard deviations. Pre-target region $\mathrm{T}-1$ includes character $\mathrm{n}-1$ and $\mathrm{n}-2$, while target region $T$ includes character $\mathrm{n}$ and $n+1$

\begin{tabular}{lccc}
\hline & Identical & Related & Unrelated \\
\hline & $\mathrm{T}-1$ & & \\
Skipping & $.16(.15)$ & $.16(.12)$ & $.17(.13)$ \\
Single fixation (ms) & $226(35)$ & $228(31)$ & $225(37)$ \\
Gaze duration (ms) & $280(64)$ & $280(47)$ & $277(58)$ \\
& $\mathrm{T}$ & & \\
Skipping & $.17(.15)$ & $.17(.12)$ & $.17(.12)$ \\
Single fixation (ms) & $235(45)$ & $255(49)$ & $261(48)$ \\
Gaze duration (ms) & $294(68)$ & $313(69)$ & $320(69)$ \\
\hline
\end{tabular}

duration, $b=15, \mathrm{SE}=6.3, p<.05$. There was no significant difference between the related and unrelated previews, $p>.1 .^{6,7}$

\section{Discussion}

The results of Experiment 1 demonstrated that reading time on the target character was longer for the related and unrelated preview conditions than for the identical preview. This result is consistent with the results of prior research (e.g., Liu et al., 2002; Tsai et al., 2004; Yang et al., 2009a) indicating that reading was slowed down when the preview was different from the target word. However, the more critical question in Experiment 1 was whether or not there were differences between the related and unrelated previews. In fact, no significant differences between these conditions were observed in either the character-based or region-based analyses.

On the surface, these results suggest that semantic information obtained from the parafovea is of no use in identifying the word when it is fixated during the reading of Chinese, consistent with the findings from reading alphabetic languages. However, this may not necessarily be the case. Since the related and unrelated previews did not fit in the sentence (i.e., they were implausible) and they were useless for understanding the text, such an implausibility effect could override the effect of semantic relatedness between the target word and the preview word. Therefore, it is necessary to exaime the semantic preview effect with plausible preview words. This was the aim of Experiment 2. In other words, the semantically unrelated and related preview words used in Experiment 2 were plausible in the sentence context. Moreover, we also examined the plausibilty of the preview word on the processing of the target word.

\footnotetext{
${ }^{6}$ Since Yan et al. (2009) reported a semantic preview benefit for integrated characters, we split the items into two groups according to the type of target words (compound or integrated characters). The majority of the target words (36 of 54) and their preview words were compound characters. No significant semantic preview benefit was observed for either kind of target word, and the pattern of results for the compound characters was identical to that based on all items.

7 To examine the effect of the predictability of the target word on the preview effects (including the typical preview benefit and the semantic preview benefit), the values of predictability norming for the target word were added as a continuous variable in the liner-mixed model. The results failed to show a main effect of predictability, or an interaction between predictability and preview effects in single fixation duration and gaze duration in both character-based and region-based analyses.
} 


\section{Experiment 2}

In Experiment 2, we examined whether there is evidence of semantic preview benefit when the semantically related and unrelated preview words fit into the sentence. Similar to Experiment 1, single character target words were used and the boundary was located at the end of character $n-1$. There were four types of previews with respect to their semantic relation to the target word and plausibility: (1) identical, (2) related \& plausible, (3) unrelated \& plausible, and (4) unrelated \& implausible. If a significant difference was observed between the related $\&$ plausible condition and the unrelated \& plausible condition, it would suggest a semantic preview benefit. Moreover, if a significant difference was found between the unrelated \& implausible condition and the other conditions (which were all plausible), it would suggest that plausibility also affects parafoveal processing during Chinese reading.

Participants. Fourty-eight undergraduate students from the South China Normal University, who did not participate in Experiment 1 participated in the experiment for course credit. They were all naïve concerning the purpose of the experiment, had normal or corrected to normal vision, and were native readers of Chinese.

Materials. Sixty experimental sentences were developed; each of them had a single-character target word embedded in the middle. In addition to the identical preview condition, three kinds of preview words were created: related \& plausible, unrelated \& plausible, and unrelated \& implausible (as discussed above). An example sentence (with the target word, related \& plausible, unrelated \& plausible, and unrelated \& implausible preview words in bold) and its English translation is:

\section{2. 陈健拎着一箱鞋/袜/桔/潭来到我经营的小店里。}

(Chen Jian brought a box of shoes/socks/oranges/ponds to my store).

Word frequency and number of strokes were matched between target words and the three types of preview words as closely as possible. The frequencies of occurrence for the target, related \& plausible, unrelated \& plausible, and unrelated \& implausible previews averaged 321 ( $\mathrm{SD}=637), 249(\mathrm{SD}=362), 226(\mathrm{SD}=318)$, and 307 ( $\mathrm{SD}=564)$ per million, with mean $\log$ frequencies of 1.96, 1.97, 1.94, and 1.97 , respectively; $F<1$. The average number of strokes for these conditions was $8.9(\mathrm{SD}=3.0), 9.2(\mathrm{SD}=3.3), 8.7(\mathrm{SD}=3.3)$ and $8.6(\mathrm{SD}=2.8), F(3,177)=1.3$, $p>.2$ Similar to Experiment 1, the non-identical preview words did not share any radicals and syllables with the target word to avoid any orthographic and homophonic benefit.

A counterbalanced design was employed in which each of the 60 sentence frames was read only once by each participant, with 15 sentences in each preview condition. In addition, 40 filler sentences were added into the material set. Each participant read 9 practice sentences followed by the 100 sentences in a random order.

Rating Studies. As in Experiment 1, a semantic relatedness study was conducted in which 18 students were asked to rate the semantic relatedness between each of the target words and the related \& plausible, unrelated \& plausible, and unrelated \& implausible preview words on a 5-point scale (where $1=$ unrelated; $5=$ highly 
related). Three counterbalanced material sets were created, each containing 20 pairs of target-related \& plausible preview words, 20 pairs of target-unrelated \& plausible preview words, and 20 pairs of target-unrelated \& implausible preview words. Participants were randomly assigned to one of these material sets. The results indicated that there was a significant main effect of semantic relatedness, $F 1$ (2, $34)=458.5, p<.001, F 2(2,118)=301.4, p<.001$. The related \& plausible preview words were more semantically related to the target words $(M=3.82$, $\mathrm{SD}=.71)$ than the unrelated \& plausible preview words $(M=1.63, \mathrm{SD}=.41)$, and the unrelated \& implausible preview words $(M=1.56, \mathrm{SD}=.53)$, ts $>19.4$, $p s<.001$. There was no significant difference between the latter two conditions, $p s>.2$.

In addition, all the experimental sentences were normed for predictability and plausibility as in Experiment 1 . In the plausibility norming study, 40 participants were presented the first part of the sentences up to (and including) the target word or its preview words and were asked to rate the plausibility of the sentence (assuming that the sentence will end with a second part) on a 5-point scale (where $1=$ highly implausible; 5 = highly plausible). Four counterbalanced material sets were created by including a fourth of the sentences in each version. The plausibility of the sentences with the target word, related \& plausible, unrelated \& plausible and unrelated \& implausible previews averaged $3.63(\mathrm{SD}=.32), 3.63(\mathrm{SD}=.34), 3.60$ $(\mathrm{SD}=.36)$, and $2.40(\mathrm{SD}=.67)$, respectively. The main effect of plausibility was significant, $F 1(3,117)=134.0, p<.001 ; F 2(3,177)=124.3, p<.001$. Pair-wise $t$ tests indicated the plausibility of sentences with the unrelated \& implausible preview word was significantly lower than the other conditions, $t \mathrm{~s}>11.8$, $p s<.001$; there were no other significant differences.

In the cloze predictability norming study, ten undergraduate students, who also rated the experimental sentences in Experiment 1 were given the first part of the experimental sentence up to (and including) the character to the left of the target word and asked to provide the next word in the sentence. Target words, the related \& plausible, and the unrelated \& plausible preview words were predicted only 2, 1 and $2 \%$ of the time, and the unrelated \& implausible preview words were never predicted.

All participants in the above rating studies were undergraduate students from the South China Normal University. They did not participant in the eye-tracking portion of Experiment 2 and they only participated in one of these rating studies.

Apparatus and Procedure. These were identical to Experiment 1.

\section{Results}

All participants averaged over $80 \%$ correct on the comprehension questions (mean $=88 \%$ ). Fixations less than $60 \mathrm{~ms}$ or greater than $600 \mathrm{~ms}$ were again eliminated from the analyses. Trials in which the display change occurred during a fixation were also excluded. In total, $13 \%$ of the data were lost (including track losses). Three contrasts were set up: (1) identical versus the average of the other three conditions, to examine the typical preview benefit, (2) unrelated \& implausible 
versus the average of the related \& plausible and unrelated \& plausible, to examine a plausibility benefit, and (3) related \& plausible versus the unrelated \& plausible, to examine a semantic preview benefit. As in Experiment 1, both character-based and region-based analyses are reported.

\section{Character-based Analysis}

The probability of initial skipping, single fixation duration, and gaze duration were computed for characters $n-1$ through character $n+1$ as a function of preview types (see Table 3). There were no differences in skipping.

Character $n-1$. A hint of a parafoveal-on-foveal effect was observed, as single fixation duration and gaze duration were longer in the identical preview condition than the average of the other three preview conditions: single fixation duration, $b=6, \mathrm{SE}=3.4, p=.065$; gaze duration, $b=9, \mathrm{SE}=3.6, p<.05$. This effect was actually due to fixation times in the unrelated \& plausible condition being shorter than in the other three conditions (which were quite comparable to each other, see Table 3). The result is puzzling as no parafoveal-on-foveal effects were observed in Experiment 1 and the pattern of fixation times on character $n-1$ (shorter times in the identical preview condition than the other conditions) was opposite to that in Experiment 2. Thus, consistent with much prior research (see Rayner et al., 2003; Rayner \& Juhasz, 2004), these potential parafoveal-on-foveal effects were not consistent across experiments (and the effect is not apparent in the region based analysis below). Furthermore, it may be that mislocalized fixations are a factor. It should also be noted that Cui, Wang, Yan, and Bai (2010) recently found that first fixation duration and gaze duration on fixated characters with high-frequent parafoveal characters were longer than those with low-frequent characters in one

Table 3 Character-based analysis: Means of eye movement measures by participants for character $n-1$ to character $\mathrm{n}+1$ in Experiment 2

\begin{tabular}{lcccc}
\hline & Identical & $\begin{array}{l}\text { Related } \\
\text { and plausible }\end{array}$ & $\begin{array}{l}\text { Unrelated } \\
\text { and plausible }\end{array}$ & $\begin{array}{l}\text { Unrelated and } \\
\text { implausible }\end{array}$ \\
\hline Skipping & $\mathrm{n}-1$ & & \\
Single fixation (ms) & $226(41)$ & $226(35)$ & $.57(.16)$ & $.52(.15)$ \\
Gaze duration (ms) & $232(46)$ & $227(34)$ & $222(34)$ & $224(34)$ \\
& $\mathrm{n}$ & & & $226(33)$ \\
Skipping & $.48(0.2)$ & $.48(0.2)$ & $.42(0.16)$ & $.49(0.2)$ \\
Single fixation (ms) & $253(36)$ & $260(45)$ & $269(46)$ & $271(55)$ \\
Gaze duration (ms) & $260(41)$ & $273(54)$ & $274(50)$ & $283(59)$ \\
Skipping & $\mathrm{n}+1$ & $.45(0.14)$ & $.48(0.15)$ & $.43(0.13)$ \\
Single fixation (ms) & $261(39)$ & $265(50)$ & $266(51)$ & $272(56)$ \\
Gaze duration (ms) & $271(44)$ & $275(53)$ & $282(55)$ & $286(54)$ \\
\hline
\end{tabular}

Values in parentheses represent standard deviations 
experiment while the direction of these effects reversed in another experiment. A similar phenomenon was also observed by Hyönä and Bertram (2004) in an alphabetic language; they found that long compound words in the parafovea yielded shorter gaze durations on the fixated word in one experiment, while long parafoveal words yielded longer gaze durations on the fixated word in a follow-up experiment.

Target word. Reading times were shorter in the identical condition than the average of the other three conditions, which reflects a typical preview benefit effect: for single fixation duration, $b=10, \mathrm{SE}=4.1, p<.05$, and gaze duration, $b=13$, $\mathrm{SE}=4.6, p<.01$. However, the effects of plausibility and semantic relatedness were not significant as the difference between the implausible condition and the two plausible preview conditions, and the difference between the related \& plausible and unrelated \& plausible condition, were not significant, $p \mathrm{~s}>.1$.

Character $n+1$. The fixation time on the spillover character was marginally shorter in the identical condition than the average of the other three conditions, for single fixation duration, $b=7, \mathrm{SE}=4.1, p=.086$, for gaze duration, $b=9$, $\mathrm{SE}=4.9, p=.079$. No other effects were significant $(p \mathrm{~s}>.2)$.

\section{Region-based analysis}

As in Experiment 1, character $\mathrm{n}-1$ and $\mathrm{n}-2$ were combined as pre-target region $\mathrm{T}-1$, and character $\mathrm{n}$ and $\mathrm{n}+1$ were combined as target region $\mathrm{T}$. This reduced the skipping rate to about $10 \%$ overall; there were no differences between the preview conditions. The probability of initial skipping, single fixation duration, and gaze duration associated with these two regions are presented in Table 4.

Pre-target Region. No significant effects were found in this region in any of the reported measures $(p s>.2)$. Thus, there was no evidence for a parafovealon-foveal effect.

Target Region. The typical preview benefit effect was reflected in shorter single fixation durations and gaze durations in the identical condition than the average of the other three conditions: single fixation duration, $b=14, \mathrm{SE}=3.6, p<.01$, gaze

Table 4 Region-based analysis: means of eye movement measures by participants for the pre-target and target region in Experiment 2

\begin{tabular}{lcccc}
\hline & Identical & $\begin{array}{l}\text { Related } \\
\text { and plausible }\end{array}$ & $\begin{array}{l}\text { Unrelated } \\
\text { and plausible }\end{array}$ & $\begin{array}{l}\text { Unrelated and } \\
\text { implausible }\end{array}$ \\
\hline Skipping & $\mathrm{T}-1$ & & & \\
Single fixation (ms) & $.15(.12)$ & $.14(.12)$ & $.18(.15)$ & $.14(.11)$ \\
Gaze duration (ms) & $225(35)$ & $228(33)$ & $221(30)$ & $220(27)$ \\
& $261(53)$ & $262(51)$ & $250(48)$ & $254(46)$ \\
Skipping & $\mathrm{T}$ & $.09(.10)$ & $.08(.09)$ & $.09(.11)$ \\
Single fixation (ms) & $265(38)$ & $271(46)$ & $283(44)$ & $293(53)$ \\
Gaze duration (ms) & $322(67)$ & $344(80)$ & $347(73)$ & $366(77)$ \\
\hline
\end{tabular}

Values in parentheses represent standard deviations. Pre-target region $\mathrm{T}-1$ includes character $\mathrm{n}-1$ and $\mathrm{n}-2$, while target region $\mathrm{T}$ includes character $\mathrm{n}$ and $\mathrm{n}+1$ 
duration, $b=23, \mathrm{SE}=5.4, p<.001$. The effect of plausibility was also significant: single fixation duration, $b=9, \mathrm{SE}=3.6, p<.01$, gaze duration, $b=14$, $\mathrm{SE}=5.6$, $p<.05$. More interestingly, a semantic preview benefit was apparent in single fixation duration (but not in gaze duration) as it was shorter in the related \& plausible condition than the unrelated \& plausible, $b=12, \mathrm{SE}=6.0, p<.05 .^{8}$

Additional contrasts were also carried out to compare the identical condition to each of the other three conditions. For single fixation duration, there was no difference between the identical condition and the related \& plausible condition $(p>.1)$. However, the identical condition yielded shorter single fixation durations than the unrelated \& plausible condition, $b=20, \mathrm{SE}=5.9, p<.001$, and the unrelated \& implausible condition, $b=28, \mathrm{SE}=6.0, p<.001$. For gaze duration, the identical condition yielded shorter durations than the other three conditions: related \& plausible, $b=23, \mathrm{SE}=8.8, p<.01$, unrelated \& plausible, $b=25$, $\mathrm{SE}=8.8, p<.01$, and unrelated \& implausible, $b=45, \mathrm{SE}=8.8, p<.001$.

\section{Supplementary analysis}

To examine why semantic preview benefit was only apparent in single fixation (and not in gaze duration) in the target region, we computed (as per Reingold, Yang, \& Rayner, 2010): (1) the probability of readers making more than one fixation on the target word (probability of a refixation), (2) the duration of the first of multiple firstpass fixations (first of multiple fixation duration), and (3) the summed duration of subsequent first-pass fixations (remainder fixation duration) on the target region.

The probability of refixation was significantly higher in the unrelated \& implausible condition (.33) than the average of the identical (.25), related \& plausible (.26), and the unrelated \& plausible ( .27$)$ conditions, $b=.25, \mathrm{SE}=.078$, $p<.01$. There were no significant differences across the four conditions in first of multiple fixation durations (although numerically there were some differences with means of 254, 257, 263, and $268 \mathrm{~ms}$ in the identical, related \& plausible, unrelated \& plausible, and unrelated \& implausible conditions, respectively). However, for the remainder fixation duration, additional contrasts revealed that the related \& plausible condition was significantly longer $(276 \mathrm{~ms})$ than the identical condition (252 ms), $b=34, \mathrm{SE}=15.8, p<.05$. The unrelated \& plausible condition and the unrelated \& implausible condition ( $259 \mathrm{~ms}$ for both) did not differ from the identical condition, $p s>.40$.

\section{Discussion}

Experiment 2 examined the effect of semantic relatedness and plausibility on preview processing with four kinds of previews: (1) identical, (2) semantically related and plausible, (3) semantically unrelated and plausible, and (4) semantically

\footnotetext{
${ }^{8}$ Again, we split the items into two groups according to the type of target words. Thus, 44 of 60 target words and their previews were compound characters, and the same sets of analyses were conducted on these items. The pattern of results based on the compound characters was similar to that based on all items, although some significant effects became marginally significant because of the loss of data points. There were not enough items to conduct analyses on the integrated characters.
} 
unrelated and implausible. Unsurprisingly, the reading time in the target word/ region was shortest in the identical condition than the other preview conditions, which is a typical preview benefit effect. Furthermore, there was a strong effect of plausibility since single fixation and gaze duration on the target region were both shorter in the plausible preview conditions than the implausible condition. However, this plausibility preview effect was not observed in the character-based analysis, which may be due to the fact that about half of the target words were skipped in first pass reading (thus reducing the reliability of this effect).

More importantly, Experiment 2 provided some evidence of a semantic preview benefit effect as single fixation duration on the target region was shorter in the related \& plausible preview condition than the unrelated \& plausible condition according to the region based analysis, which is in contrast to the null effects in Experiment 1. It should be noted that the manipulation of semantic relatedness between the two experiments was different: while the related and unrelated preview words in Experiment 1 were implausible, they were plausible in Experiment 2. It seems that semantic preview benefit in Chinese can only be obtained with plausible preview words.

But, the fact that the semantic preview benefit effect disappeared in gaze duration indicates that the amount of preview benefit from a related preview word is attenuated when Chinese readers make more than one fixation on the target region. The pattern of data wherein an effect was observed in single (or first) fixation duration but not in gaze duration is not typical, but it is also not without precedence. For example, Pollatsek, Lesch, Morris, and Rayner (1992) observed such a pattern in a study dealing with phonological preview benefit and, more recently, Rayner, Castelhano, and Yang (2010) observed a preview benefit effect in older readers on first fixation that disappeared in gaze duration. In the latter study, it was found that older readers were much more likely to refixate the target word prior to making a saccade to another word and that first of multiple fixation duration and remainder fixation duration were longer for the older readers than the younger readers across all conditions (including the identical preview condition). Thus, the preview benefit effect was attenuated for older readers in gaze duration. Rayner et al. concluded that on most trials $(80 \%)$ older readers obtain preview benefit that is comparable to younger readers, but on the remaining trials the preview benefit is attenuated because their processing is slower and they need to refixate the word to fully process it.

In the present study, we obtained effects that are comparable to those reported by Rayner et al. (2010) in the sense that preview benefit was evident in single fixation duration but not gaze duration. However, unlike Rayner et al., we did not find differences due to the number of refixations and we failed to find any effects of preview manipulation in first of multiple fixation durations. However, we did find differences in the remainder fixation durations. Thus, when readers were able to process the target word with a single fixation (which happened $72 \%$ of the time), a related \& plausible preview word provided facilitation for the processing of the target word. However, when they had to make more than one fixation before leaving the target word, a related \& plausible preview word yielded longer remainder fixation durations than an identical preview word, while first of multiple fixation 
durations did not differ across conditions. We suspect that this pattern of data can be explained in the following way. Given that it is more efficient for Chinese readers to process the target word with a single fixation than with multiple fixations, related \& plausible preview words help to activate the meaning of the target word (thus yielding a semantic preview benefit). Nevertheless, in the case when readers made multiple fixations, the integration of semantic information across fixations is delayed (due to less efficient processing) yielding no effects in first of multiple fixation durations. However, foveal processing of the target word continues, although it may be less efficient as compared to when the target word received only one fixation. Therefore, at the later part of the foveal processing of the target word (which is reflected by the remainder fixation durations), the related \& plausible preview word competes with the target word as a lexical candidate. Because the two words have overlapping semantic information, there is an interference effect. Basically, we take the single fixation duration data at face value and suspect that Chinese readers are sometimes able to obtain semantic information from a word before fixating on it.

\section{General discussion}

In the experiments reported here, we examined whether Chinese readers obtain semantic information from the word to the right of fixation and then use it for identifying the word when it is later fixated. In Experiment 1, the semantic relation between the preview and the target word was manipulated via three kinds of previews: identical, semantically related, and unrelated. Neither the related nor the unrelated previews were plausible in the sentence. The results revealed that reading times on the target word were not different between the related previews and the unrelated previews, although both of them were longer than the identical previews. In Experiment 2, semantically related and unrelated preview words were plausible in the sentence, to test whether there is a semantic preview benefit effect when the preview word is potentially of use for understanding the text. Thus, there were four conditions in the experiment: (1) identical preview, (2) related \& plausible preview, (3) unrelated \& plausible preview, and (4) unrelated \& implausible preview. Results from the region-based analysis indicated that readers fixated longer on the target word in the unrelated \& implausible preview condition than the average of the related \& plausible and unrelated \& plausible conditions, suggesting a plausibility preview benefit. Moreover, there was a difference between the related \& plausible and unrelated \& plausible preview conditions in single fixation duration in the target region. Taken together, these results suggest an interaction between the effect of semantic relatedness and plausibility of a preview word on the processing of a target word: a semantic preview benefit was only apparent when the preview words were plausible. The results also suggest that the effect of plausibility is stronger than the effect of semantic relatedness during the reading of Chinese.

It is important to note that most previous studies on semantic parafoveal coding focused only on the semantic relation between the preview and the target word (Rayner \& Morris, 1992; Rayner et al., 1986; Altarriba et al., 2001) as in our 
manipulation in Experiment 1. The results from the reading of alphabetic languages were consistent with our findings in Experiment 1 in that related parafoveal words did not provide preview benefit for later processing of the target word. However, Yan et al. (2009) recently reported a semantic preview benefit effect in Chinese reading. In their experiment, there were five kinds of preview characters for the target characters: (1) identical, (2) orthographically related, (3) phonologically related, (4) semantically related, and (5) unrelated. A reliable benefit was found from orthographically and semantically related previews (phonological preview benefit was marginally significant in gaze duration). Thus, unlike our results, Yan et al. obtained a semantic preview benefit effect even though the semantically related preview word was not plausible in the sentence context.

The inconsistent results across Yan et al.'s study and our studies could be due to stimulus differences. While targets in Yan et al.'s study were integrated characters, and each of them was embedded as the first character of a two-character word in the sentence, targets in our study were single-character nouns and most of them were compound characters. As noted by Yan et al., the integrated target characters in their experiment were mapped more closely to meaning than to phonology, and thus maximized chances of observing a semantic preview benefit effect. Moreover, compared with our target characters, Yan et al.'s target characters were highly frequent (averaging over 1,000 per million vs. about 250 per million for ours), and their visual complexity was less complicated (the average number of strokes was 5 vs. 9 for ours). The lighter processing load associated with their target characters may also be a factor in obtaining a semantic preview benefit effect in Chinese. Thus, further studies are needed to confirm to what extent the preview effect from semantically related previews depends on the layout of a character (integrated or compound), visual complexity, frequency, and whether it is a single-character word or a component character of a multiple-character word.

Although semantically related parafoveal characters did not provide preview benefit for the target characters in our Experiment 1, it is not necessarily the case that semantic information from a preview character is of no use for the processing of that character when it is later fixated. In Experiment 2, we not only observed evidence for semantic preview effects, more importantly, we found preview benefit from a plausible preview word compared to an implausible preview word, which suggests that semantic interpretation occurs in the parafovea to some extent.

The finding that contextual effects on word recognition occur at a very early stage, even before the word is fixated, is consistent with the fact that readers fixate for less time on high-predictable target words than on low-predictable target words and they are less likely to fixate on high-predictable target words than on lowpredictable target words; this finding holds for readers of alphabetic writing systems (Rayner \& Well, 1996; Rayner, Ashby, Pollatsek, \& Reichle, 2004; Brysbaert \& Vitu, 1998) and Chinese (Rayner, Li, Juhasz, \& Yan, 2005; Wang, Pomplun, Chen, Ko, \& Rayner, 2010). Moreover, Balota et al. (1985) found that readers obtain larger preview benefit from high-predictable target words than low-predictable target words. In one of their experimental sentences "Since the wedding was today, the baker rushed the wedding cake/pies to the reception", cake was the highpredictable target word and pies was the low-predictable target word. Each target 
word had four kinds of previews: visually similar, semantically related, visually dissimilar, and anomalous, which were cahc, pies, picz, and bomb, respectively, for the target word cake, and picz, cake, cahc, and bomb, respectively for the target word pies. The results showed robust preview benefits from the visually similar previews, which was larger for high-predictable target words than low-predictable target words.

However, in Balota et al's study, a highly predictable preview provided no facilitation for low predictable target words, and vice versa. That is, fixation times on the target word (cake or pies) were not significantly different between the semantic preview (pies or cake for the high- and low-predictable target words, respectively) and the anomalous preview (bomb), which suggested semantic information from parafoveal words has no effect on identifying the target word (see also Drieghe, Rayner, \& Pollatsek, 2005; Rayner et al., 1986). These results again suggest that the semantic and plausibility preview benefit observed in the current study could be a language-specific phenomenon that doesn't generalize to the reading of alphabetic languages, like English. As emphasized in the introduction, the distinctive characteristic of written Chinese (in comparison to English) is that there are no explicit marks between words, and thus Chinese readers have to rely on contextual information to segment words on-line. In addition, given that more information (relatively speaking) falls within the foveal region in Chinese than in English, Chinese readers could be more effective in using information to the right of fixation. Consistent with this hypothesis, there is evidence that characters $n+1$ (the character immediately following the target character) and $n+2$ are processed during a fixation on the target character n (Yang et al., 2009b; Yan, Kliegl, Shu, Pan, \& Zhou, 2010).

In conclusion, Chinese readers show deeper semantic processing to the right of fixation than English readers. They not only obtain semantic information from the word to the right of fixation, but they also integrate this word with the context, which is reflected by the robust influence from the plausibility of a preview word on the processing of the target word. Moreover, the preview effect of plausibility seems stronger than preview effects due to semantic relatedness.

Acknowledgments The research in this article was supported by Grants from the National Natural Science Foundation of China (30970894), the Guangdong Natural Science Foundation (06200524), the Program for New Century Excellent Talent in Universities in China (No. NCET-08-0645), and Grant HD26765 from the (US) National Institute of Health. Portions of these experiments were presented at the Third China International Conference on Eye Movements, Zhuhai, China, June, 2008.

Open Access This article is distributed under the terms of the Creative Commons Attribution Noncommercial License which permits any noncommercial use, distribution, and reproduction in any medium, provided the original author(s) and source are credited.

\section{References}

Altarriba, J., Kambe, G., Pollatsek, A., \& Rayner, K. (2001). Semantic codes are not used in integrating information across eye fixations in reading: Evidence from fluent Spanish-English bilinguals. Perception \& Psychophysics, 63, 875-890. 
Ashby, J., \& Rayner, K. (2004). Representing syllable information during silent reading: Evidence from eye movements. Language and Cognitive Processes, 19, 391-426.

Ashby, J., Treiman, R., Kessler, B., \& Rayner, K. (2006). Vowel processing during silent reading: Evidence from eye movements. Journal of Experimental Psychology. Learning, Memory, and Cognition, 32, 416-424.

Baayen, R. H. (2008). Analyzing linguistic data: A practical introduction to statistics. Cambridge, UK: Cambridge University Press.

Baayen, R. H., Davidson, D. H., \& Bates, D. M. (2008). Mixed-effects modeling with crossed random effects for subjects and items. Journal of Memory and Language, 59, 390-412.

Balota, D. A., Pollatsek, A., \& Rayner, K. (1985). The interaction of contextual constraints and parafoveal visual information in reading. Cognitive Psychology, 17, 364-390.

Bates, D.M., \& Maechler, M. (2009). lme4: Linear mixed-effect models using S4 classes. R package version 0.999375-1.

Briihl, D., \& Inhoff, A. W. (1995). Integrating information across fixations during reading: The use of orthographic bodies and of exterior letters. Journal of Experimental Psychology. Learning, Memory, and Cognition, 21, 55-67.

Brysbaert, M., \& Vitu, F. (1998). Word skipping: Implications for theories of eye movement control in reading. In G. Underwood (Ed.), Eye guidance in reading and scene perception (pp. 125-147). Amsterdam: Elsevier.

Chace, K. H., Rayner, K., \& Well, A. D. (2005). Eye movements and phonological preview benefit: Effects of reading skill. Canadian Journal of Experimental Psychology, 59, 209-217.

Chen, H.-C., Flores d'Arcais, G. B., \& Cheung, S. L. (1995). Orthographic and phonological activation in recognizing Chinese characters. Psychological Research, 58, 144-153.

Chen, H.-C., \& Shu, H. (2001). Lexical activation during the recognition of Chinese characters. Psychonomic Bulletin \& Review, 8, 511-518.

Chen, H.-C., Song, H., Lau, W. Y., Wong, K. F. E., \& Tang, S. L. (2003). Developmental characteristics of eye movements in reading Chinese. In C. McBride-Chang \& H.-C. Chen (Eds.), Reading development in Chinese children (pp. 157-169). Westport, CT: Praeger.

Cui, L., Wang, S., Yan, G., \& Bai, X. (2010). Parafoveal-on-foveal interactions in Chinese normal reading (Chinese). Acta Psychologica Sinica, 42, 547-558.

Drieghe, D., Rayner, K., \& Pollatsek, A. (2005). Eye movements and word skipping during reading revisited. Journal of Experimental Psychology: Human Perception and Performance, 31, 954-969.

Drieghe, D., Rayner, K., \& Pollatsek, A. (2008). Mislocated fixations can account for parafovealon-foveal effects in eye movements during reading. Quarterly Journal of Experimental Psychology, $61,1239-1249$.

Henderson, J. M., Dixon, P., Petersen, A., Twilley, L. C., \& Ferreira, F. (1995). Evidence for the use of phonological representations during transsaccadic word recognition. Journal of Experimental Psychology: Human Perception and Performance, 21, 82-97.

Hyönä, J., \& Bertram, R. (2004). Do frequency characteristics of non-fixated words influence the processing of fixated words during reading? European Journal of Cognitive Psychology, 16, 104-127.

Hyönä, J., \& Häikiö, T. (2005). Is emotional content obtained from parafoveal words during reading? An eye movement analysis. Scandinavian Journal of Psychology, 46, 475-483.

Inhoff, A. W. (1989). Parafoveal processing of words and saccade computation during eye fixations in reading. Journal of Experimental Psychology: Human Perception and Performance, 15, 544-555.

Inhoff, A. W. (1990). Integrating information across eye fixations in reading: The role of letter and word units. Acta Psychologica, 73, 281-297.

Inhoff, A. W., \& Liu, W. (1997). The perceptual span during the reading of Chinese text. In H.-C. Chen (Ed.), The cognitive processing of Chinese and related Asian languages. Hong Kong: The Chinese University Press.

Inhoff, A. W., \& Liu, W. (1998). The perceptual span and oculomotor activity during the reading of Chinese sentences. Journal of Experimental Psychology: Human Perception and Performance, 24, 20-34.

Inhoff, A. W., Radach, R., Eiter, B. M., \& Juhasz, B. (2003). Distinct subsystems for the parafoveal processing of spatial and linguistic information during eye fixations in reading. Quarterly Journal of Experimental Psychology: Human Experimental Psychology, 56, 803-827.

Juhasz, B. J., White, S. J., Liversedge, S. P., \& Rayner, K. (2008). Eye movements and the use of parafoveal word length information in reading. Journal of Experimental Psychology: Human Perception and Performance, 34, 1560-1579. 
Lima, S. D. (1987). Morphological analysis in sentence reading. Journal of Memory and Language, 26, 84-99.

Lima, S. D., \& Inhoff, A. W. (1985). Lexical access during eye fixations in reading: Effect of word-initial letter sequences. Journal of Experimental Psychology: Human Perception and Performance, 11, 272-285.

Liu, W., Inhoff, A. W., Ye, Y., \& Wu, C. (2002). Use of parafoveally visible characters during the reading of Chinese sentences. Journal of Experimental Psychology: Human Perception and Performance, 28, 1213-1227.

Miellet, S., \& Sparrow, L. (2004). Phonological codes are assembled before word fixation: Evidence from boundary paradigm in sentence reading. Brain and Language, 90, 299-310.

Morris, R. K., Rayner, K., \& Pollatsek, A. (1990). Eye movement guidance in reading: The role of parafoveal letter and space information. Journal of Experimental Psychology: Human Perception and Performance, 16, 268-281.

Pollatsek, A., Lesch, M., Morris, R. K., \& Rayner, K. (1992). Phonological codes are used in integrating information across saccades in word identification and reading. Journal of Experimental Psychology: Human Perception and Performance, 18, 148-162.

Rayner, K. (1975). The perceptual span and peripheral cues in reading. Cognitive Psychology, 7, 65-81.

Rayner, K. (1998). Eye movements in reading and information processing: 20 years of research. Psychological Bulletin, 124(3), 372-422.

Rayner, K. (2009). The Thirty Fifth Sir Frederick Bartlett Lecture: Eye movements and attention during reading, scene perception, and visual search. Quarterly Journal of Experimental Psychology, 62, 1457-1506.

Rayner, K., Ashby, J., Pollatsek, A., \& Reichle, E. D. (2004). The effects of frequency and predictability on eye fixations in reading: Implications for the E-Z Reader model. Journal of Experimental Psychology: Human Perception and Performance, 30, 720-732.

Rayner, K., Balota, D. A., \& Pollatsek, A. (1986). Against parafoveal semantic preprocessing during eye fixations in reading. Canadian Journal of Psychology, 40, 473-483.

Rayner, K., \& Juhasz, B. J. (2004). Eye movements in reading: Old questions and new directions. European Journal of Cognitive Psychology, 16, 340-352.

Rayner, K., Li, X., Juhasz, B. J., \& Yan, G. (2005). The effect of word predictability on the eye movements of Chinese readers. Psychonomic Bulletin \& Review, 12, 1089-1093.

Rayner, K., \& Morris, R. K. (1992). Eye movement control in reading: Evidence against semantic preprocessing. Journal of Experimental Psychology: Human Perception and Performance, 18, $163-172$.

Rayner, K., \& Well, A. D. (1996). Effects of contextual constraint on eye movements in reading: A further examination. Psychonomic Bulletin \& Review, 3, 504-509.

Rayner, K., Castelhano, M.S., \& Yang, J. (2010). Preview benefit during eye fixations in reading for older and younger readers. Psychology and Aging, 25(3), 714-718.

Rayner, K., White, S. J., Kambe, G., Miller, B., \& Liversedge, S. P. (2003). On the processing of meaning from parafoveal vision during eye fixation in reading. In J. Hyönä, R. Radach, \& H. Deubel (Eds.), The mind's eye: Cognitive and applied aspects of eye movements (pp. 213-234). Amsterdam: Elsevier Science.

Reingold, E. M., Yang, J., \& Rayner, K. (2010). The time course of word frequency and case alternation effects on fixation times in reading: Evidence for lexical control of eye movements. Journal of Experimental Psychology: Human Perception and Performance (in press).

Starr, M. S., \& Rayner, K. (2001). Eye movements during reading: Some current controversies. Trends in Cognitive Sciences, 5, 156-163.

R Development Core Team (2009). R: A language and environment for statistical computing. R Foundation for Statistical Computing, Vienna, Austria. ISBN 3-900051-07-0, URL http://www. R-project.org.

Tsai, J.-L., Lee, C.-Y., Tzeng, O. J., Hung, D. L., \& Yen, N.-S. (2004). Use of phonological codes for Chinese characters: Evidence from processing of parafoveal preview when reading sentences. Brain and Language, 91, 235-244.

Wang, S., Chen, H.-C., Yang, J., \& Mo, L. (2008). Immediacy of integration in discourse comprehension: Evidence from Chinese readers' eye movements. Language and Cognitive Processes, 23, 241-257.

Wang, H.-C., Pomplun, M., Chen, M., Ko, H., \& Rayner, K. (2010). Estimating the effect of word predictability on eye movements in Chinese reading using latent semantic analysis and transitional probability. Quarterly Journal of Experimental Psychology (in press). 
White, S. J., Bertram, R., \& Hyönä, J. (2008). Semantic processing of previews within compound words. Journal of Experimental Psychology. Learning, Memory, and Cognition, 34, 988-993.

$\mathrm{Xu}$, Y., Pollatsek, A., \& Potter, M. C. (1999). The activation of phonology during silent Chinese word reading. Journal of Experimental Psychology. Learning, Memory, and Cognition, 25, 838-857.

Yan, M., Kliegl, R., Shu, H., Pan, J., \& Zhou, X. (2010). Parafoveal load of word N + 1 modulates preprocessing effectiveness of word $\mathrm{N}+2$ in Chinese reading. Journal of Experimental Psychology: Human Perception and Performance (in press).

Yan, M., Richter, E., Shu, H., \& Kliegl, R. (2009). Readers of Chinese extract semantic information from parafoveal words. Psychonomic Bulletin \& Review, 16, 561-566.

Yang, J., Wang, S., Chen, H.-C., \& Rayner, K. (2009a). The time course of semantic and syntactic information processing in Chinese sentence comprehension: Evidence from eye movements. Memory \& Cognition, 37, 1164-1176.

Yang, J., Wang, S., Xu, Y., \& Rayner, K. (2009b). Do Chinese readers obtain preview benefit from character $\mathrm{n}+2$ ? Evidence from eye movements. Journal of Experimental Psychology: Human Perception and Performance, 35, 1192-1204.

Yin, B. (1984). A quantitative research of Chinese morphemes. Zhongguo Yuwen, 5, 338-347. 